Check for updates

Cite this: RSC Adv., 2017, 7, 26710

Received 13th January 2017

Accepted 27th March 2017

DOI: $10.1039 / c 7 r a 00547 d$

rsc.li/rsc-advances

\section{Substrate-mediated growth of vanadium carbide with controllable structure as high performance electrocatalysts for dye-sensitized solar cells $\uparrow$}

\begin{abstract}
Jutao Jin, (D) ${ }^{a}$ Zhiyang Wei, ${ }^{b}$ Xiaochang Qiao, (D) ${ }^{a}$ Hongbo Fan ${ }^{\star a}$ and Lifeng Cui ${ }^{\star a}$
Transition metal carbides (TMCS) are promising alternatives to noble-metal based catalysts in many fields. Controlling the morphology of TMCs is an effective approach to promote the catalytic performance. We here report a substrate-mediated method to direct the growth of vanadium carbide (VC) using graphene oxide as the nucleation and growth substrate. By carefully choosing the synthesis conditions, we can tune the structure of VC nanoparticles from cuboctahedron (VC-ch) to cubic (VC-cb). The obtained hybrid of VC-ch on graphene sheets (VC-ch/GS) shows excellent catalytic activity towards the triiodide reduction reaction in terms of the charge transfer resistance $\left(R_{\mathrm{ct}}=0.27 \Omega \mathrm{cm}^{2}\right)$, outperforming its counterpart VC-cb/GS $\left(R_{\mathrm{ct}}=1.69 \Omega \mathrm{cm}^{2}\right)$. The energy conversion efficiency of the Dye-Sensitized Solar Cells (DSSC) with the VC-ch counter electrode (CE) reached $7.92 \%$, comparable to a Pt based CE of 7.79\%. Experimental combined with theoretical calculation results suggest that the high catalytic activity of VC-ch may originate from the special atom packing state of the $\mathrm{VC}(111)$ facet.
\end{abstract}

\section{Introduction}

Due to their high efficiency, low cost and simple fabrication, dye-sensitized solar cells (DSSCs) have attracted considerable attention during the past two decades. ${ }^{1-4}$ The main technological hurdle to the development of DSSCs with the $\mathrm{I}_{3}{ }^{-} / \mathrm{I}^{-}$redox shuttle is the sluggish kinetics of the $\mathrm{I}_{3}{ }^{-}$reduction to $\mathrm{I}^{-}$at the counter electrode (CE)/electrolyte interface, ${ }^{5}$ which requires the exclusive use of platinum-based catalysts. ${ }^{5-7}$ The expensive and scarce nature of platinum has hindered its large scale applications. Therefore, extensive efforts have been dedicated to improving the performance of platinum based catalysts, and also to seek alternative catalysts based on abundant and nonprecious materials. ${ }^{6,8-22}$

Early transitional metal carbides (TMCs) are promising alternatives to noble-metal based catalysts in many fields, such as dehydrogenation, ammonia decomposition and also as electrocatalysts. ${ }^{18,23}$ Levy first reported the Pt-like properties of tungsten carbides for catalyzing the oxidation of hydrogen in the presence of oxygen at room temperature. They found that carbon atoms in WC can modify the electronic structure of $\mathrm{W}$ to give rise to a Pt-like d-band electronic structure. ${ }^{24}$ Since then the catalytic properties of TMCs have been the subject of many investigations in the fields of

${ }^{a}$ Dongguan University of Technology, School of Environment and Civil Engineering, China.E-mail:fhb@dgut.edu.cn; lifeng.cui@gmail.com

${ }^{b}$ Institute of Physics, Chinese Academy of Sciences, China

$\dagger$ Electronic supplementary information (ESI) available. See DOI: 10.1039/c7ra00547d catalysis and surface science. The potential applications of TMCs as counter electrode electrocatalysts for DSSCs has been also intensively studied, recently. ${ }^{18,25,26}$ Lately, Wu etc. systematically investigated the performances of three classes (carbides, nitrides and oxides) of nanoscaled early-transitionmetal as potential catalysts for DSSCs. They found that the DSSC using the in situ formed vanadium carbide (VC) embedded in mesoporous carbons as the counter electrode outperformed other transitional metal carbides, nitrides and oxides based DSSCs. ${ }^{18} \mathrm{VC}$, an early transitional metal carbide (TMC), often shows face-centered cubic structure with the carbon atoms occupying the interstitial spaces between vanadium metal atoms. The bonding in VC involves simultaneous contributions from metallic $(\mathrm{V}-\mathrm{V})$, covalent $(\mathrm{C}-\mathrm{C})$, and ionic $(\mathrm{V}-\mathrm{C})$ bonding. ${ }^{23}$ Specially, VC show strong shapedependent catalytic performances. This can be ascribed to their substantially different atomic packing structure at different crystalline planes, e.g. the $\mathrm{VC}(100)$ surface is characterized by the coexistence of the metal and carbon atoms, while in the (111) orientation, it has alternating layers of metal and carbon atoms, leading to the (111) surface either terminated by pure metal or by pure carbon..$^{23}$ Recently, the shape controlled synthesis of nanoparticles including metal and metal oxides nanocrystal, has made great achievements. ${ }^{27-33}$ Nevertheless, the synthesis of VC particles with well-defined morphology is rarely reported. ${ }^{34-36}$ Traditionally, VC particles are synthesized by directly thermally annealing transitional metal oxides or metal salts with carbon containing precursors. ${ }^{37,38}$ Unfortunately, the morphology is difficult to control and the particle size is mainly in 
micrometer scale for VC synthesized with such method. Therefore, exploring facile methods for synthesis of VC with controllable crystalline facets to explore their structure-performance relationships is of great importance for developing highly qualified TMC-based electrocatalysts.

Herein, we report a substrate-mediated method to direct the growth of VC using graphene oxide as the nucleation and growth substrate. By simply tuning the precursor ratio of $\mathrm{VOCl}_{3}$ to cyanamide, we can controllably synthesize cubic (VC-cb) and cuboctahedral (VC-ch) vanadium carbide nanoparticles, respectively. These nanosized VC nanoparticles were used as counter electrode catalysts for triiodide reduction reaction, and showed comparable catalytic activity to noble Pt based ones. Specially, VC particles show morphology depending catalytic activity towards triiodide reduction reaction, in which $\mathrm{VC}$ nanoparticles with cuboctahedral shape show superior catalytic activity to the cubic one.

\section{Experiment section}

\section{Materials synthesis}

GO was synthesized by a modified Hummers method. To

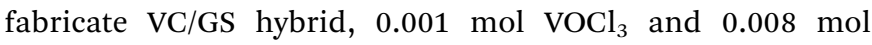
cyanamide $\left(\mathrm{CN}_{2} \mathrm{H}_{2}\right)$ was added in proper sequence to $100 \mathrm{mg}$ GO dispersed in $90 \mathrm{~mL}$ ethanol and $10 \mathrm{~mL}$ deionized water and the mixture was followed by magnet stirring at $80^{\circ} \mathrm{C}$ for $8 \mathrm{~h}$. Then the reaction solution was transferred into glassware and heated at $60{ }^{\circ} \mathrm{C}$ until a slurry was formed. The slurry was further dried by freeze-drying. Finally the production was heated to $1100{ }^{\circ} \mathrm{C}$ for $3 \mathrm{~h}$ at a rate of $3{ }^{\circ} \mathrm{C} \mathrm{min}^{-1}$ under argon atmosphere to synthesize VC-ch/GS hybrid. By changing the cyanamide content to $0.012 \mathrm{~mol}$, the same method leaded to the VC-cb/GS hybrid. VC nanoparticles were also synthesized by the exact same method as above without adding graphene oxide.

\section{Electrochemical measurements}

$10 \mathrm{mg}$ VC-ch/GS or VC-cb/GS hybrid catalyst was dispersed in 3 $\mathrm{mL} 0.1 \mathrm{wt} \%$ Nafion solution by sonication for more than $1 \mathrm{~h}$ to form homogeneous ink. To assemble the counter electrode (CE), $60 \mu \mathrm{L}$ of the catalyst ink was loaded onto a pre-cleaned FTO glass and the efficiency area was fixed to $1 \mathrm{~cm}^{2}$. Thus, the loading was about $0.20 \mathrm{mg} \mathrm{cm}^{-2}$. To prepare Pt CEs, $20 \mu \mathrm{L}$ of $5 \mathrm{mM} \mathrm{H}_{2} \mathrm{PtCl}_{6}$ in isopropanol was drop casted on a pre-cleaned $1.5 \mathrm{~cm}^{2} \mathrm{FTO}$ glass, followed by heating at $380^{\circ} \mathrm{C}$ for $30 \mathrm{~min}$. The symmetrical dummy cells used in the electrochemical impedance spectroscopy (EIS) experiments and the Tafel-polarization test were conducted with two identical CEs sealed by a $60 \mu \mathrm{m}$ Surlyn 1702 film. The active area of the dummy cell was 0.25 $\mathrm{cm}^{2}$. The electrolyte used was the same as that used in DSSCs

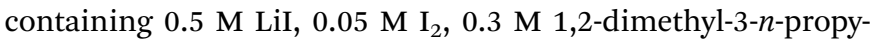
limidazolium iodide and $0.5 \mathrm{M}$ 4-tert-butyl pyridine anhydrous acetonitrile. The Tafel polarization measurements were performed at a scanning rate of $50 \mathrm{mV} \mathrm{s}^{-1}$. For EIS experiments, the cells were scanned from $0.1 \mathrm{~Hz}$ to $100 \mathrm{kHz}$ at $0 \mathrm{~V}$ bias and AC amplitude of $10 \mathrm{mV}$.

\section{Fabrication of DSSCs}

The photoanode of DSSCs was prepared by a method reported previously. ${ }^{25} \mathrm{~A}$ mixture of $1 \mathrm{~g} \mathrm{\textrm {TiO } _ { 2 }}$ powder, $2.0 \mathrm{~mL} \mathrm{H}_{2} \mathrm{O}, 30 \mu \mathrm{L}$ acetylacetone and $20 \mu \mathrm{L}$ Triton X-100 was coated on a FTO glass by a doctor blade technique, followed by annealing at $500{ }^{\circ} \mathrm{C}$ for $40 \mathrm{~min}$ in atmosphere. After cooling to $80^{\circ} \mathrm{C}$, the $\mathrm{TiO}_{2}$ anode was immersed in a solution containing 0.5 mM N719 dye (Solaronix) in a mixed solvent of acetonitrile and isobutanol (v/v $=1 / 1$ ) for $24 \mathrm{~h}$ to form the sensitized photoanode.

\section{Characterization}

X-ray diffraction (XRD) measurements were carried out using an automatic X-ray powder diffractometer (D/Max 2400, Rigaku). Transmission electron microscopy (TEM) was performed on a Tecnai G2-F30 with an accelerating voltage of $300 \mathrm{kV}$. XPS spectrum was collected on a VG Scientific ESCALAB210photoelectron spectrometer with a $\mathrm{Mg} \mathrm{K} \alpha \mathrm{X}$-ray resource. The EIS measurement and Tafel polarization measurement were performed on an Autolab workstation. The photocurrent density-voltage curves of DSSCs were measured by a Keithley 2635 Souremeter under AM $1.5 \mathrm{G}$ illumination $\left(100 \mathrm{~mW} \mathrm{~cm}^{-2}\right)$ using a solar simulator (Newport).

\section{Computational details}

First-principles calculations were carried out based on density functional theories using the Vienna Ab initio Simulation Package (VASP). ${ }^{39}$ The exchange and correlation potentials were calculated by using the PW91 form for generalized gradient approximation (GGA). ${ }^{40}$ We used a plane-wave basis set and the projector augmented wave (PAW) potentials. ${ }^{41}$ An energy cutoff of $400 \mathrm{eV}$ was chosen to ensure the convergence of total groundstate energy. The Brillouin zone of the super cell was sampled by $6 \times 6 \times 1$ points within the Monkhorst-Pack scheme. Electronic energies were computed with the SCF tolerance of $10^{-5} \mathrm{eV}$ and total forces were converged to less than $0.02 \mathrm{eV} \AA^{-1}$.

The I-binding energies for the as-investigated vanadium carbide were evaluated through equation:

$$
E_{\mathrm{ad}}(\mathrm{I})=E(\mathrm{VC}-\mathrm{I})-1 / 2 E\left(\mathrm{I}_{2}\right)-E(\mathrm{VC})
$$

where $E_{\mathrm{ad}}(\mathrm{I})$ is the I-binding energy, $E(\mathrm{VC}-\mathrm{I})$ is the total energy of the I adduct, $E\left(\mathrm{I}_{2}\right)$ is the energy of the isolated $\mathrm{I}_{2}$ molecule, and $E(\mathrm{VC})$ is the energy of $\mathrm{VC}$ vacuum interface.

\section{Results and discussion}

The morphology and microstructure of the samples were characterized and analyzed. Fig. 1a is the TEM image of the typical sample synthesized with a cyanamide/ $\mathrm{VOCl}_{3}$ (abbreviated as $\mathrm{C} / \mathrm{V}$ ) precursors at $8: 1$, which shows homogeneously dispersed nanoparticles with mainly cuboctahedron-shaped morphology on GS. Interestingly, these supported nanoparticles were transformed to cubic-dominated morphology by adjusting the molar ratio of $\mathrm{C} / \mathrm{V}$ to $12: 1$ (Fig. 1b). In a controlled experiment, the exactly same synthesis procedure was conducted but without adding $\mathrm{GO}$ in the starting materials. 



Fig. 1 VC nanoparticles on graphene sheets (GS) in VC/GS hybrids. (a and b) TEM images of (a) the cuboctahedral VC nanoparticles on graphene sheet (VC-ch/GS) and (b) the cubic VC nanoparticles on graphene sheet (VC-cb/GS), respectively. The insert shows high-resolution TEM image of VC nanoparticles with a distance of (a) $0.241 \mathrm{~nm}$ and (b) $0.208 \mathrm{~nm}$ between adjacent planes, corresponding to the $d$-spacing of (111) and (200) planes, respectively. The inserts at top left corners of (a) and (b) shows selected area electron diffraction (SAED) of the corresponding hybrids. (c) XRD patterns and (d) $\mathrm{N}_{2}$ adsorption/desorption isotherms of VC-ch/GS hybrid and VC-cb/GS hybrid.

In contrast, much larger aggregated VC nanoparticles with irregular shape formed in the absence of GO (Fig. $\mathrm{S} 1 ; \dagger$ around 50-150 nm for nominal $\mathrm{C} / \mathrm{V}$ atomic ratio of $\sim 8$; around $30-$ $80 \mathrm{~nm}$ for nominal $\mathrm{C} / \mathrm{V}$ atomic ratio of $\sim 12$ ). These results demonstrate that GO plays an important role for growth of regular VC particles during the synthesis process. Here, GO can adsorb the $\mathrm{V}$ ions with its surface defects and functional groups to mediate the formation of intermediate $\mathrm{VO}_{x}$ products at first. Then these homogeneously dispersed $\mathrm{VO}_{x}$ particles are transformed to VC particles in carbonic atmosphere created by the $\mathrm{CN}_{2} \mathrm{H}_{2}$ precursor during the high temperature carbonization process. The ratio of precursor $\mathrm{GO}$ to $\mathrm{VOCl}_{3}$ is also important to the formation of homogeneously dispersed VC nanoparticles on GS. A high ratio of $\mathrm{VOCl}_{3}$ to $\mathrm{GO}$ in the solution as leads to the aggregated VC particles on GS, while a low ratio of $\mathrm{VOCl}_{3}$ to GO leads to sparse VC particles on GS (Fig. S2 $\dagger$ ). The XRD patterns of both products confirm the presence of $\mathrm{VC}$ with a crystal structure well matched with the corresponding JCPDS data (no. 65-8818). The selected area electron diffraction (SAED) patterns also confirm a face-centered cubic crystal structure of both samples (insert of Fig. 1a and b). High-resolution TEM images show well crystal structure of these nanoparticles with clear lattice fingers. Brunauer-Emmett-Teller (BET) measurements show that the specific surface area of VC-cb/GS hybrid and VCch/GS hybrid is 242.15 and $200.76 \mathrm{~m}^{2} \mathrm{~g}^{-1}$ (Fig. 1d), respectively, much higher than those metal carbide or nitride materials synthesized by "Urea Glass" route. ${ }^{\mathbf{1 8 3 8 , 4 2}}$ The chemical composition of both samples were characterized with X-ray photoelectron spectroscopy (XPS), showing the presence of $\mathrm{C}$, N, V, O in both VC-cb/GS and VC-ch/GS hybrids (Fig. S3†).

As observed above, the morphology of the supported $\mathrm{VC}$ particles is sensitive to the molar ratio of $\mathrm{C} / \mathrm{V}$ precursor. In fact, under thermodynamic control, the seed crystal should take on the shape with minimized surface energy during their growth process. In our case, the synthesized VC is a high symmetric face-centered cubic crystal, and the surface energy follows an energy sequence of $\gamma_{\{111\}}<\gamma_{\{100\}}<\gamma_{\{110\}}$. According to the Wulff's theorem, the seed crystal should take on a truncated octahedron morphology with 6(100) and 8(111) exposed at the crystal surface for a face centered cubic crystal. ${ }^{43}$ As a matter of fact, the (100) and (111) facets have intrinsic different atomic coordination environment. The (111) are kinked facets that have abundant kinks at the surface, while the (100) facet is a flat surface. ${ }^{32,44}$ At lower $\mathrm{CN}_{2} \mathrm{H}_{2}$ proportion (the molecular ratio of $\mathrm{CN}_{2} \mathrm{H}_{2}$ to $\mathrm{VOCl}_{3}$ is 8 ), the pyrolyzed products of $\mathrm{CN}_{2} \mathrm{H}_{2}$ only create poor carbonic atmosphere around VC. The crystal growth is controlled by the affordable carbonic free radicals, thus the (100) and (111) facets have almost equivalent growth rate, leading to cuboctahedral nanoparticles with 6(100) facets and $8(111)$ facets. While at higher $\mathrm{CN}_{2} \mathrm{H}_{2}$ proportion (the molecular ratio of $\mathrm{CN}_{2} \mathrm{H}_{2}$ to $\mathrm{VOCl}_{3}$ is 12 ), the carbonic free radicals around the VC crystal are abundant. The crystal growth rate in this case is controlled by the adsorption ability of the facet. The (111) facets with rich kinks at the surface have more chance to absorb the incoming atoms and grow faster. Thus, the VC nanoparticles finally end up with cubic shape. 
The DSSCs with VC-ch/GS, VC-cb/GS and Pt based CEs were fabricated to evaluate their catalytic performance. The loading amount of different catalysts on CEs was fixed equally to each other for reasonable comparison. The anode of the DSSCs is FTO glass deposited with $\mathrm{TiO}_{2}$ nanoparticles sensitized by cisdiisothiocyanato-bis(2,2'-bipyridyl-4,4'-dicarboxylato)ruthenium(II)bis(tetrabutylammonium) (N719) dye. Fig. 2 shows photocurrent density-voltage $(J-V)$ curve measured under AM 1.5 illumination of DSSCs with various CEs. As shown in Table 1 , the Pt-based reference DSSC yields an open circuit voltage $\left(V_{\mathrm{oc}}\right)$ of $0.71 \pm 0.02 \mathrm{~V}$, a short circuit current $\left(J_{\mathrm{sc}}\right)$ of $15.21 \pm 0.26$ $\mathrm{mA} \mathrm{cm} \mathrm{cm}^{-2}$, a fill factor (FF) of $68.87 \pm 0.38 \%$, and an overall power conversion efficiency of $7.58 \pm 0.05 \%$. The DSSC with the VC-ch/GS CE exhibited a $V_{\text {oc }}$ of $0.69 \pm 0.02 \mathrm{~V}$, a remarkable $J_{\mathrm{sc}}$ of $16.71 \pm 0.23 \mathrm{~mA} \mathrm{~cm}^{-2}$, and a higher FF of $72.12 \pm 0.35 \%$, and a power conversion efficiency of $7.83 \pm 0.05 \%$. In contrast, the DSSC with VC-cb/GS CE produced the $V_{\text {oc }}, J_{\text {sc }}, \mathrm{FF}$, and power conversion efficiency were only $0.69 \pm 0.01 \mathrm{~V}, 15.48 \pm 0.35 \mathrm{~mA}$ $\mathrm{cm}^{-2}, 62.01 \pm 0.45 \%$, and $6.48 \pm 0.08 \%$, respectively. These results clearly demonstrate the morphology dependence of the VC based CEs for DSSCs. To the best of our knowledge, this is the first report of morphology-dependent triiodide reduction reaction electrocatalytic properties for $\mathrm{VC}$ nanoparticles. In addition, the photovoltaic performance of the DSSCs using VC nanoparticles without GS as CEs were also investigated (Fig. S4†). The relatively inferior photovoltaic parameters of the VC nanoparticles based CEs compared to those of the corresponding VC/GS hybrids suggests a synergic effect between the VC nanoparticles and GS.

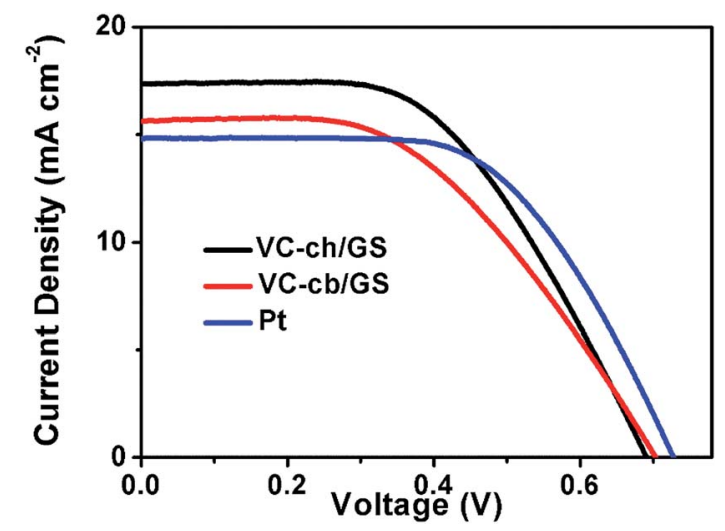

Fig. 2 Photocurrent density-voltage curves of DSSCs with the different CEs, measured under standard AM 1.5 G illumination $(100 \mathrm{~mW}$ $\mathrm{cm}^{-2}$ ).

Table 1 Photovoltaic characteristics of DSSCs with various CEs

\begin{tabular}{lllll}
\hline Sample & $V_{\text {oc }}$ & $J_{\text {sc }}\left(\mathrm{mA} \mathrm{cm}^{-2}\right)$ & FF (\%) & Efficiency (\%) \\
\hline VC-ch/GS & $0.69 \pm 0.02$ & $16.71 \pm 0.23$ & $72.12 \pm 0.35$ & $7.83 \pm 0.05$ \\
VC-cb/GS & $0.69 \pm 0.01$ & $15.48 \pm 0.35$ & $62.01 \pm 0.45$ & $6.48 \pm 0.08$ \\
VC8 & $0.69 \pm 0.02$ & $13.20 \pm 0.18$ & $59.32 \pm 0.68$ & $5.43 \pm 0.07$ \\
VC12 & $0.68 \pm 0.01$ & $14.50 \pm 0.30$ & $55.71 \pm 0.39$ & $5.32 \pm 0.08$ \\
Pt & $0.71 \pm 0.02$ & $15.21 \pm 0.26$ & $68.87 \pm 0.38$ & $7.58 \pm 0.05$
\end{tabular}

To investigate the electrochemical activities of the as-prepared hybrids on the reduction of triiodide $\left(\mathrm{I}_{3}{ }^{-}\right)$, electrochemical impedance spectroscopic (EIS) of the DSSCs with VC-ch/GS, VCcb/GS based CEs were carried out, respectively. For comparison, Pt-coated FTO glass CE was measured as a reference point (see Experimental section for fabrication details). As seen from the obtained Nyquist plots (Fig. 3a), the absolute series resistance $\left(R_{\mathrm{s}}\right)$, corresponding to high-frequency intercept on the real axis, retains almost the same for all the three CEs, which is consistent with the results from four-probe measurement (Table S1 $\dagger$ ). While the charge transfer resistance $\left(R_{\mathrm{ct}}\right)$, simulated from the semicircle in the high-frequency region of the Nyquist plot based on a circuit model (Fig. S5 in ESI†), varies significantly for the three CEs. The $R_{\text {ct }}$ value of VC-ch/GS is $0.27 \Omega \mathrm{cm}^{2}$, much lower than that of Pt CE $\left(0.52 \Omega \mathrm{cm}^{2}\right)$, suggesting its excellent catalytic activity for triiodide reduction reaction. In contrast, $R_{\mathrm{ct}}$ of $\mathrm{VC}-\mathrm{cb} /$ GS $\left(1.69 \Omega \mathrm{cm}^{2}\right)$ is much higher than those of Pt and VC-ch/GS. These results further indicate that the catalytic performance of VC nanoparticles toward triiodide reduction reaction is highly shape-dependent. The cuboctahedral VC-ch shows obviously higher catalytic activity than the cubic one. This phenomenon is further confirmed by the Tafel polarization curves. The exchange current density $\left(J_{0}\right)$, reflecting the inherent catalytic property of a catalyst, can be calculated from the extrapolated intercepts of the anodic and cathodic branches of the polarization curve $(J-V$ plot). Higher Tafel slope indicates higher exchange current density and higher catalytic activity. ${ }^{17}$ Among the three CEs, VCch/GS hybrid possessed the highest Tafel slope and then Pt, while VC-cb/GS showed the lowest slope (Fig. 3b). This result is
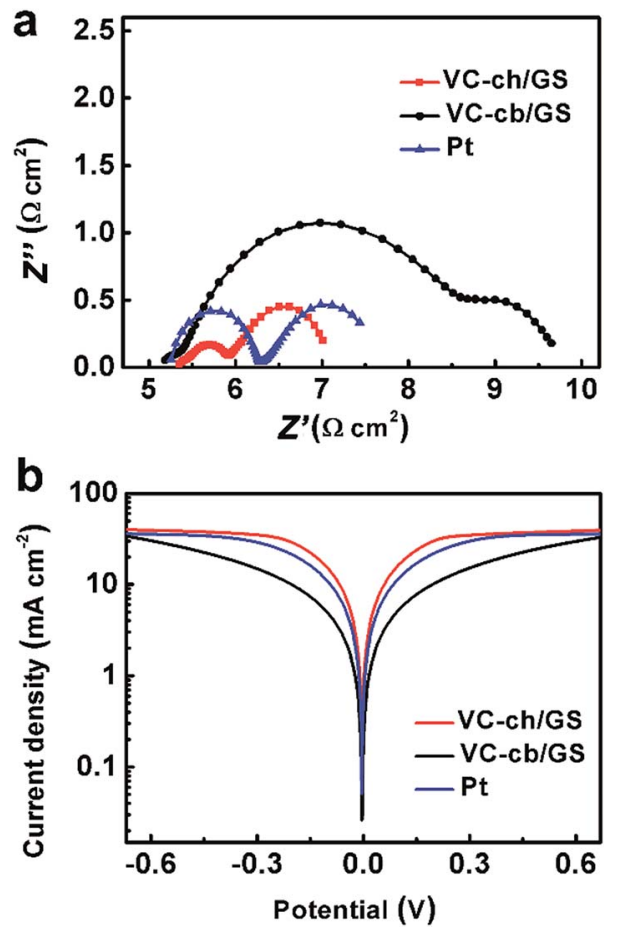

Fig. 3 (a) Nyquist plots and (b) corresponding Tafel polarization curves of the symmetric dummy cells fabricated by VC-ch/GS hybrid, VC-cb/ GS hybrid and Pt counter electrodes (CEs). 
consistent with the EIS analysis that VC-ch/GS possesses higher catalytic activity than VC-cb/GS and Pt. Interestingly, at the diffusion region of the polarization curve (e.g., the high overpotential region), the limiting diffusion current $\left(J_{\text {lim }}\right)$ of the three CEs is nearly equal (Fig. 3b), which could be easily understood by noticing that the three CEs have almost the same diffusion coefficient illustrated by the similar diameters of semicircles in the low-frequency region of the Nyquist plot (Fig. 3a). These results demonstrate that the catalytic difference between the VC$\mathrm{ch} / \mathrm{GS}$ and VC-cb/GS is resulted from the different crystalline facets rather than the diffusion ability of $\mathrm{I}_{3}{ }^{-} / \mathrm{I}^{-}$at the electrolyte/ electrode surface. Furthermore, the fact that the specific surface area of VC-cb/GS is a little higher than that of VC-ch/GS hybrid suggests the excellent catalytic activity of VC-ch/GS originates from the higher specific activity of a single active site rather than from more available active site numbers (the standard methods for the test of electrochemical active surface area is not available now). Further experiments confirm that either VC nanoparticles or NG exhibits inferior catalytic performance compared to the corresponding VC/GS hybrid (Fig. S6 and S7†), indicating synergistic triiodide reduction reaction catalytic activity of VC and GS in the hybrid. The GS in VC/GS hybrid can act as an interconnected conducting network to form an electron transport pathway. In general, triiodide $\left(\mathrm{I}_{3}{ }^{-}\right)$reduction reaction proceeds on the $\mathrm{CE}$ through multisteps reactions involving, the decomposition of $\mathrm{I}_{3}{ }^{-}$in solution to $\mathrm{I}_{2}$ and $\mathrm{I}^{-}$through a fast process, the subsequent formation of intermediate absorbed I on active site through $\mathrm{I}_{2}$ dissociation at the liquid-solid interface, following by its further one-electron reduction to $\mathrm{I}^{-}$ions. Both theoretical and experimental evidences have confirmed that the last step, which involves the absorption and desorption of $\mathrm{I}$ is the rate-determinestep. Catalysis of triiodide reduction reaction strongly depends on the absorption affinity for I on the catalytic active sites.

To reveal the differences in intrinsic catalytic activities of VCch/GS and VC-cb/GS hybrid towards triiodide reduction reaction, we conducted high resolution XPS analyses. The V $2 \mathrm{p}_{3 / 2}$ band can be deconvoluted into three bands at 513.5, 515.1 and $517.1 \mathrm{eV}$ (Fig. 4a), assigned to $\mathrm{V}$ atoms as $\mathrm{V}-\mathrm{V}, \mathrm{V}-\mathrm{O}$ and $\mathrm{V}-\mathrm{C}$ structure, respectively. The $\mathrm{V}-\mathrm{O}$ bonds result from the surface absorption of oxygen molecular, thus its binding energy is lower than that of $\mathrm{V}-\mathrm{C}$ bonds. For VC-cb/GS sample, the $\mathrm{C}-\mathrm{V}$ bond is found to be the dominant component in all of the three types of $\mathrm{V}$ states, while the $\mathrm{V}-\mathrm{V}$ and $\mathrm{V}-\mathrm{O}$ are negligible. Upon VC-ch/ GS, the band corresponding to $\mathrm{V}-\mathrm{C}$ becomes weaker, while the bands belong to $\mathrm{V}-\mathrm{V}$ and $\mathrm{V}-\mathrm{O}$ become much stronger. The difference of the surface chemical state of $\mathrm{V}$ in the two hybrids can be explained by the different exposed facets of VC in the two hybrids. As mentioned above, the cuboctahedral VC particle is enclosed by $6\{100\}$ facets and $8\{111\}$ facets, while the cubic VC particle is enclosed by $6\{100\}$ facets. As shown in Fig. 4 b, the surface $\mathrm{V}$ atoms at (100) facet is coordinated with five $\mathrm{C}$ atoms, similar to the body $\mathrm{V}$ atoms enclosed by six $\mathrm{C}$ atoms. Thus the $\mathrm{V}$ atom in (100) facet shows similar oxidation as the body one. As for $\mathrm{V}$ atoms in (111) facet, each $\mathrm{V}$ atom is coordinated with three $\mathrm{C}$ atoms at sub-layer and six in-layer $\mathrm{V}$ atoms. Clearly, the $\mathrm{V}$ atoms at (111) facet possess metal-like chemical state, which is consistent with the XPS result. In fact, the alternating layers of cations (C) and anions (V) in the [111] direction lead to a polar surface, which is highly beneficial for the adsorption of small molecular like I ions. ${ }^{45}$ The result can explain the presence of the strong $\mathrm{V}-\mathrm{O}$ band of $\mathrm{VC}-\mathrm{ch} / \mathrm{GS}$ in XPS results. We proposed that the polar surface may also benefit for the adsorption of I atoms at electrode/solution surface during the electrocatalysis process.

To gain deep insight into the morphology dependent triiodide reduction reaction process on VC samples, we conducted DFT calculations (see details in Experimental section) to study I adsorption on the two different exposed surface, VC(111) and VC(100). For comparison, Pt(100) surface was also calculated. The calculated results suggest that the VC(111) surface can bond I atom much stronger than that of VC(100), even surpass that of $\mathrm{Pt}(100)$. Fig. 5 shows the charge density difference map of the adsorption state of single I atom on VC(111) and VC(100), respectively. It can be seen clearly that the electrons transfer from surface of $\mathrm{V}$ atom to I atom, leading to the electrons accumulate at I and depletion at the surface $\mathrm{V}$ atom for both VC(111) and VC(100) surfaces. In comparison with $\mathrm{VC}(100)$ surfaces, VC(111) surface shows an obvious orbital overlap between d-orbital of surface $\mathrm{V}$ and the p-orbital of $\mathrm{I}$ atom, indicating a strengthened $\mathrm{V}-\mathrm{I}$ bond on $\mathrm{VC}(111)$ compared that
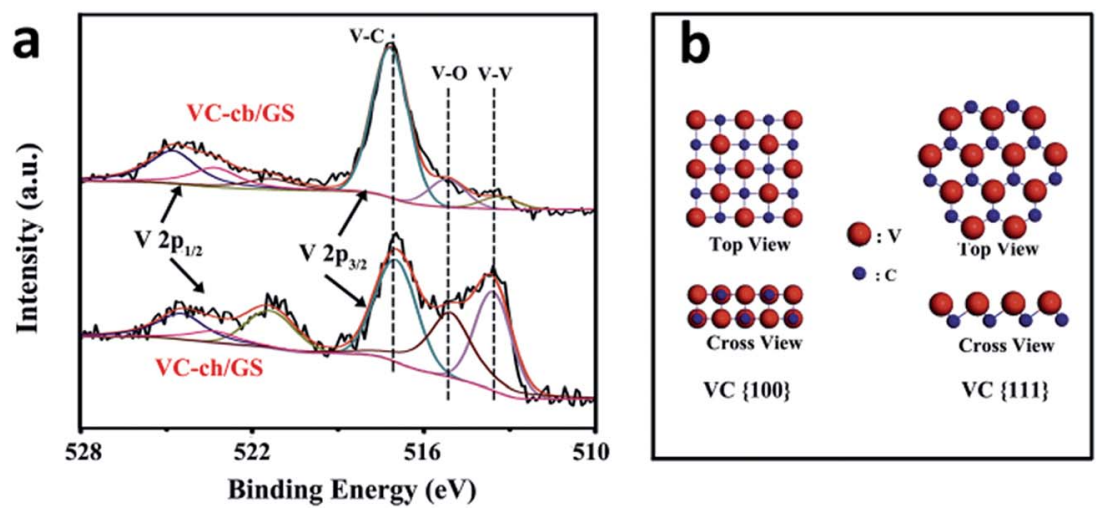

Fig. 4 (a) High resolution X-ray photoelectron spectroscopy (XPS) of VC-ch/GS hybrid and VC-cb/GS hybrid. (b) Schematic diagram of surface atomic arrangement of VC(100) and VC(111) facets. 
a

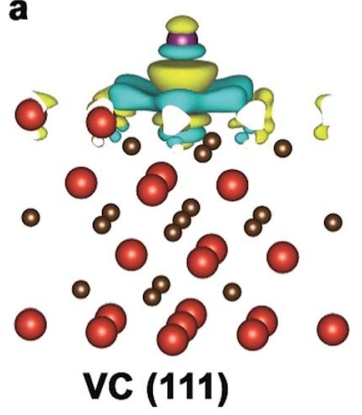

b

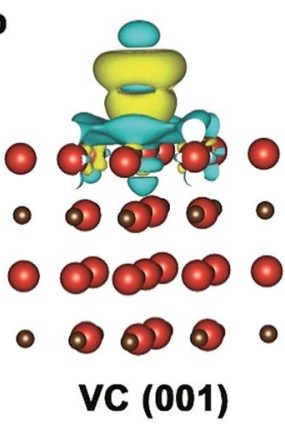

Fig. 5 Charge density difference map of the adsorption state of single I atom on VC(111) (a) and $\mathrm{VC}(100)$ (b), yellow color represents for electron accumulation while light-blue for electron depletion.

of $\mathrm{VC}(100)$. The $\mathrm{VC}(111)$ surface shows a V-I binding energy of $0.6 \mathrm{eV}$, approaching the optimum binding energy $(0.5 \mathrm{eV})$ for the catalyze the triiodide reduction reaction. While for $\mathrm{VC}(100)$ surface, it shows a V-I binding energy of $0.3 \mathrm{eV}$, which may be too weak to the catalytic process.

\section{Conclusions}

In conclusion, $\mathrm{VC}$ nanoparticles with controllable morphology have been successfully synthesized via a graphene mediated high temperature annealing method. By changing the precursor ratio, we can tune the morphology of VC from cuboctahedral to cubic shape. Significantly, the VC nanoparticles show shape dependent catalytic properties towards triiodide reduction reaction, where VC with cuboctahedral shape shows superior performance to the cubic one. We propose that the excellent catalytic activity of VC-ch may originate from the highly efficient (111) facet, which affords a suitable absorption energy towards the reactants and products. The DSSCs with VC-ch based CEs yielded a power conversion efficiency of $7.92 \%$, slightly higher than $7.79 \%$ of Pt based catalysts. Our experiment results indicated that manipulating the shape of TMCs can offer a great opportunity to design high performance electrocatalysts for various chemical reactions in renewable energy field.

\section{Author contributions}

The manuscript was written through contributions of all authors. All authors have given approval to the final version of the manuscript.

\section{Acknowledgements}

This work was financially supported by the National Nature Science Foundation (NSFC) of China under award no. 21403227, DICP Fundamental Research Program for Clean Energy (2013D102), Chinese Postdoctoral Science Foundation 2014M551136.

\section{References}

1 B. O'regan and M. Grfitzeli, A low-cost, high-efficiency solar cell based on dye-sensitized, Nature, 1991, 353, 737-740.
2 A. Hagfeldt, G. Boschloo, L. Sun, L. Kloo and H. Pettersson, Dye-Sensitized Solar Cells, Chem. Rev., 2010, 110, 6595-6663.

3 H. Pettersson, K. Nonomura, L. Kloo and A. Hagfeldt, Trends in patent applications for dye-sensitized solar cells, Energy Environ. Sci., 2012, 5, 7376-7380.

4 S. Zhang, X. Yang, Y. Numata and L. Han, Highly efficient dye-sensitized solar cells: progress and future challenges, Energy Environ. Sci., 2013, 6, 1443-1464.

5 A. Listorti, B. O'Regan and J. R. Durrant, Electron Transfer Dynamics in Dye-Sensitized Solar Cells, Chem. Mater., 2011, 23, 3381-3399.

6 B. Zhang, D. Wang, Y. Hou, S. Yang, X. H. Yang, J. H. Zhong, J. Liu, H. F. Wang, P. Hu and H. J. Zhao, Facet-dependent catalytic activity of platinum nanocrystals for triiodide reduction in dye-sensitized solar cells, Sci. Rep., 2013, 3, 1836-1842.

7 V.-D. Dao, S. H. Ko, H.-S. Choi and J.-K. Lee, Pt-NP-MWNT nanohybrid as a robust and low-cost counter electrode material for dye-sensitized solar cells, J. Mater. Chem., 2012, 22, 14023-14029.

8 Y. Liao, K. Pan, L. Wang, Q. Pan, W. Zhou, X. Miao, B. Jiang, C. Tian, G. Tian, G. Wang and H. Fu, Facile Synthesis of High-Crystallinity Graphitic Carbon/Fe3C Nanocomposites As Counter Electrodes for High-Efficiency Dye-Sensitized Solar Cells, ACS Appl. Mater. Interfaces, 2013, 5, 3663-3670.

9 C.-W. Kung, H.-W. Chen, C.-Y. Lin, K.-C. Huang, R. Vittal and K.-C. Ho, CoS Acicular Nanorod Arrays for the Counter Electrode of an Efficient Dye-Sensitized Solar Cell., ACS Nano, 2012, 6, 7016-7025.

10 Z. Wen, S. Cui, H. Pu, S. Mao, K. Yu, X. Feng and J. Chen, Metal nitride/graphene nanohybrids: general synthesis and multifunctional titanium nitride/graphene electrocatalyst, Adv. Mater., 2011, 23, 5445-5450.

11 Y.-C. Wang, D.-Y. Wang, Y.-T. Jiang, H.-A. Chen, C.-C. Chen, K.-C. Ho, H.-L. Chou and C.-W. Chen, FeS2 Nanocrystal Ink as a Catalytic Electrode for Dye-Sensitized Solar Cells, Angew. Chem., Int. Ed., 2013, 52, 6694-6698.

$12 \mathrm{M}$. Wu, X. Lin, A. Hagfeldt and T. Ma, Low-Cost Molybdenum Carbide and Tungsten Carbide Counter Electrodes for Dye-Sensitized Solar Cells, Angew. Chem., Int. Ed., 2011, 50, 3520-3524.

13 X. Lin, M. Wu, Y. Wang, A. Hagfeldt and T. Ma, Novel counter electrode catalysts of niobium oxides supersede Pt for dyesensitized solar cells, Chem. Commun., 2011, 47, 11489-11491.

14 E. Ramasamy, C. Jo, A. Anthonysamy, I. Jeong, J. K. Kim and J. Lee, Soft-Template Simple Synthesis of Ordered Mesoporous Titanium Nitride-Carbon Nanocomposite for High Performance Dye-Sensitized Solar Cell Counter Electrodes, Chem. Mater., 2012, 24, 1575-1582.

15 J. He, N. W. Duffy, J. M. Pringle and Y.-B. Cheng, Conducting polymer and titanium carbide-based nanocomposites as efficient counter electrodes for dye-sensitized solar cells, Electrochim. Acta, 2013, 105, 275-281.

16 F. Gong, H. Wang, X. Xu, G. Zhou and Z.-S. Wang, In situ growth of Co0. 85Se and Ni0. 85Se on conductive substrates as high-performance counter electrodes for dye- 
sensitized solar cells, J. Am. Chem. Soc., 2012, 134, 1095310958.

17 M. Wang, A. M. Anghel, B. Marsan, N.-L. Cevey Ha, N. Pootrakulchote, S. M. Zakeeruddin and M. Grätzel, CoS Supersedes Pt as Efficient Electrocatalyst for Triiodide Reduction in Dye-Sensitized Solar Cells, J. Am. Chem. Soc., 2009, 131, 15976-15977.

18 M. Wu, X. Lin, Y. Wang, L. Wang, W. Guo, D. Qi, X. Peng, A. Hagfeldt, M. Grätzel and T. Ma, Economical Pt-Free Catalysts for Counter Electrodes of Dye-Sensitized Solar Cells, J. Am. Chem. Soc., 2012, 134, 3419-3428.

19 Y. Hou, D. Wang, X. H. Yang, W. Q. Fang, B. Zhang, H. F. Wang, G. Z. Lu, P. Hu, H. J. Zhao and H. G. Yang, Rational screening low-cost counter electrodes for dyesensitized solar cells, Nat. Commun., 2013, 4, 1583.

20 X. Xu, D. Huang, K. Cao, M. Wang, S. M. Zakeeruddin and M. Grätzel, Electrochemically Reduced Graphene Oxide Multilayer Films as Efficient Counter Electrode for DyeSensitized Solar Cells, Sci. Rep., 2013, 3, 1489-1495.

21 S. Peng, J. Shi, J. Pei, Y. Liang, F. Cheng, J. Liang and J. Chen, Ni1 $-x$ Pt $x(x=0-0.08)$ films as the photocathode of dyesensitized solar cells with high efficiency, Nano Res., 2009, 2, 484-492.

22 X. Wang, Y. Xie, B. Bateer, K. Pan, Y. Zhou, Y. Zhang, G. Wang, W. Zhou and H. Fu, Hexagonal FeS nanosheets with high-energy (001) facets: counter electrode materials superior to platinum for dye-sensitized solar cells, Nano Res., 2016, 1-13.

23 H. H. Hwu and J. G. Chen, Surface Chemistry of Transition Metal Carbides, Chem. Rev., 2004, 105, 185-212.

24 R. B. Levy and M. Boudart, Platinum-Like Behavior of Tungsten Carbide in Surface Catalysis, Science, 1973, 181, 547-549.

$25 \mathrm{M}$. Wu, X. Lin, A. Hagfeldt and T. Ma, Low-Cost Molybdenum Carbide and Tungsten Carbide Counter Electrodes for Dye-Sensitized Solar Cells, Angew. Chem., Int. Ed., 2011, 50, 3520-3524.

26 J. He, N. W. Duffy, J. M. Pringle and Y.-B. Cheng, Conducting polymer and titanium carbide-based nanocomposites as efficient counter electrodes for dye-sensitized solar cells, Electrochim. Acta, 2013, 105, 275-281.

27 H. Zhang, M. Jin, Y. Xiong, B. Lim and Y. Xia, Shapecontrolled synthesis of Pd nanocrystals and their catalytic applications, Acc. Chem. Res., 2012, 46, 1783-1794.

28 Y. Xia, Y. Xiong, B. Lim and S. E. Skrabalak, ShapeControlled Synthesis of Metal Nanocrystals: Simple Chemistry Meets Complex Physics?, Angew. Chem., Int. Ed., 2009, 48, 60-103.

29 J. Zhang, H. Yang, B. Martens, Z. Luo, D. Xu, Y. Wang, S. Zou and J. Fang, Pt-Cu nanoctahedra: synthesis and comparative study with nanocubes on their electrochemical catalytic performance, Chem. Sci., 2012, 3, 3302-3306.
30 T. Teranishi and M. Miyake, Size Control of Palladium Nanoparticles and Their Crystal Structures, Chem. Mater., 1998, 10, 594-600.

31 Y.-X. Chen, S.-P. Chen, Z.-Y. Zhou, N. Tian, Y.-X. Jiang, S.-G. Sun, Y. Ding and Z. L. Wang, Tuning the shape and catalytic activity of $\mathrm{Fe}$ nanocrystals from rhombic dodecahedra and tetragonal bipyramids to cubes by electrochemistry, J. Am. Chem. Soc., 2009, 131, 10860-10862.

32 J. E. Millstone, W. Wei, M. R. Jones, H. Yoo and C. A. Mirkin, Iodide ions control seed-mediated growth of anisotropic gold nanoparticles, Nano Lett., 2008, 8, 2526-2529.

33 Y. Sun and Y. Xia, Shape-controlled synthesis of gold and silver nanoparticles, Science, 2002, 298, 2176-2179.

34 J. G. Chen, Carbide and Nitride Overlayers on Early Transition Metal Surfaces: Preparation, Characterization, and Reactivities, Chem. Rev., 1996, 96, 1477-1498.

35 D. E. Grove, U. Gupta and A. Castleman Jr, Effect of carbon concentration on changing the morphology of titanium carbide nanoparticles from cubic to cuboctahedron, ACS Nano, 2009, 4, 49-54.

36 D. E. Grove, U. Gupta and A. W. Castleman, Effect of Hydrocarbons on the Morphology of Synthesized Niobium Carbide Nanoparticles, Langmuir, 2010, 26, 16517-16521.

37 P. Li, M. Lei and W. Tang, Route to transition metal carbide nanoparticles through cyanamide and metal oxides, Mater. Res. Bull., 2008, 43, 3621-3626.

38 C. Giordano, C. Erpen, W. Yao and M. Antonietti, Synthesis of Mo and $\mathrm{W}$ carbide and nitride nanoparticles via a simple "urea glass" route, Nano Lett., 2008, 8, 4659-4663.

39 G. Kresse and J. Furthmüller, Efficient iterative schemes for ab initio total-energy calculations using a plane-wave basis set, Phys. Rev. B: Condens. Matter Mater. Phys., 1996, 54, 11169-11186.

40 J. P. Perdew and Y. Wang, Accurate and simple analytic representation of the electron-gas correlation energy, Phys. Rev. B: Condens. Matter Mater. Phys., 1992, 45, 13244-13249.

41 G. Kresse and D. Joubert, From ultrasoft pseudopotentials to the projector augmented-wave method, Phys. Rev. B: Condens. Matter Mater. Phys., 1999, 59, 1758-1775.

42 C. Giordano, C. Erpen, W. Yao, B. Milke and M. Antonietti, Metal Nitride and Metal Carbide Nanoparticles by a Soft Urea Pathway, Chem. Mater., 2009, 21, 5136-5144.

43 B. Lee and S. Komarneni, Chemical processing of ceramics, CRC Press, 2010.

44 N. Radenović, W. van Enckevort and E. Vlieg, Formamide adsorption and habit changes of alkali halide crystals grown from solutions, J. Cryst. Growth, 2004, 263, 544-551.

45 J. R. Kitchin, J. K. Nørskov, M. A. Barteau and J. G. Chen, Trends in the chemical properties of early transition metal carbide surfaces: a density functional study, Catal. Today, 2005, 105, 66-73. 